\title{
Vulnerabilidad de los estudiantes de comunicación social. Un estudio de caso ${ }^{1}$
}

\author{
Recibido: 27 de marzo de 2019 \\ Aceptado: 3 de mayo de 2019 \\ Publicado: 24 de junio de 2019
}

\author{
Piedad Ruiz Echeverry \\ pruiz@unicauca.edu.co \\ Universidad del Cauca (Colombia)
}

Resumen: El artículo parte de una investigación terminada en 2018, cuyo objetivo fue identificar las situaciones y causas de vulnerabilidad física, emocional, social y económica de los estudiantes del Programa de Comunicación social. En procura de ello se recogió información de los estudiantes a través de cuatro grupos de discusión y 125 encuestas cada una con 49 preguntas cerradas, lo cual constituyó el insumo para el análisis. Los resultados de la investigación permitieron identificar algunas características socioeconómicas como que el estudiante promedio del Programa es residente en barrios de estrato medio-bajo, no consume tres alimentos completos al día y depende de su familia para pagar su matrícula y su sustento. También que la pertenencia a la población LGBTIQ+, la maternidad o paternidad, así como las dificultades financieras son las condiciones de mayor vulnerabilidad estudiantil e inciden en la calidad de vida del entorno formativo y en el proceso mismo de formación.

Palabras clave: Caracterización, vulnerabilidad, discriminación, formación, estudiantes, comunicación social.

\begin{abstract}
This article start with a research completed in 2018, whose goal was to identify situations and causes of physical, emotional, social and economic vulnerability of the students of the Social Communication's program. In pursuit of this aim, four discussion groups, 125 surveys shaped with 49 closed questions each, provided the information for the analysis. The results of the research, enabled the identification of some socioeconomic characteristics of the students, such as that the program average student lives in low-middle class neighborhoods, does not get to have three proper meals a day and depends on his family to pay their student fees and their sustenance. The research also showed that belonging to the LGBTIQ+ population, parenting, and financial difficulties, represent some bigger student vulnerability conditions which impact in the quality of the training environment and the forming process itself.
\end{abstract}

Key words: Characterization, Vulnerability, Discrimination, Training Students, Social Communication.

1 Proyecto "Casos y causas de vulnerabilidad estudiantil en el Programa de Comunicación Social de la Universidad del Cauca”, del Grupo de Investigaciones y Estudios de Comunicación (Universidad del Cauca). Resultados presentados como ponencia en el I Congreso Nacional de Comunicación de AFACOM, Bogotá (Colombia), el 20 de marzo de 2019. 


\section{Introducción}

El presente artículo da cuenta de una parte de los resultados de la investigación desarrollada durante el segundo semestre de 2017, derivada de algunos requerimientos identificados en el Plan de Mejoramiento del Programa de Comunicación Social de la Universidad del Cauca en Colombia (PCSUC) (Unicauca, 2017a).

El proceso de autoevaluación realizado con fines de acreditación de alta calidad había permitido identificar previamente el siguiente problema, que se concibió como una oportunidad de mejora: no existe información precisa, clara y actualizada sobre casos y causas de vulnerabilidad física, emocional, social y económica de los estudiantes del PCSUC, ni un protocolo de seguimiento y atención a los casos de vulnerabilidad, tanto para evitar la deserción e inasistencia como para promover que los estudiantes tengan una mejor calidad de vida en el entorno universitario.

Por lo anterior, se decidió realizar una investigación ${ }^{2}$, cuyo objetivo general fue identificar las situaciones y causas de vulnerabilidad física, emocional, social y económica de los estudiantes del PCSUC, que afectan su interés en el proceso formativo, rendimiento, asistencia a clase, permanencia y graduación, como insumo para el diseño de un protocolo de seguimiento y atención permanente, que incorpore estrategias de atención durante el proceso formativo por parte de los docentes, de intervención técnica por parte de la Facultad y de asistencia integral por parte de la Vicerrectoría de cultura y bienestar.

Como objetivos específicos se definieron los siguientes:

- Identificar los casos de estudiantes del PCSUC en condición de vulnerabilidad y las causas de la misma.

- Diseñar un protocolo de evaluación periódica y atención de la vulnerabilidad de los estudiantes del PCSUC, a partir del seguimiento de los casos de inasistencia, retiro y repitencia.

- Divulgar los resultados ante las entidades universitarias pertinentes, para el diseño de estrategias y herramientas de seguimiento y atención estudiantil complementarias, a nivel institucional.

Antes de revisar algunos antecedentes, es fundamental tener en cuenta algunos elementos contextuales. El PCSUC cumple 20 años de funcionamiento en el 2019 y está adscrito a la Facultad de Derecho, Ciencias Políticas y Sociales, contando en los últimos años con un promedio de 243 estudiantes matriculados semestralmente. La Universidad del Cauca es una institución pública de educación superior, con 190 años de existencia. Para el último semestre de 2018, contó con 17.422 estudiantes en sus 63 programas de pregrado (Unicauca, 2018).

2 Radicada en la Vicerrectoría de Investigaciones de la Universidad del Cauca, con el ID 4583. 
Además de atender demanda educativa en el propio departamento del Cauca, ejerce una atracción importante de estudiantes en 6 departamentos circunvecinos de la zona suroccidental de la geografía colombiana. Una característica especial del contexto cultural y social de la universidad, es que el departamento del Cauca cuenta con varias comunidades indígenas que habitan resguardos en zonas rurales, así como con comunidades afrodescendientes asentadas en territorios colectivos, lo cual representa un escenario pluriétnico y multicultural. Adicionalmente, la población estudiantil de la universidad proviene mayoritariamente de zonas urbanas con poblaciones menores a 500000 habitantes y de zonas rurales del departamento del Cauca y otros departamentos cercanos.

Adicionalmente, es imprescindible tener en cuenta que el departamento del Cauca ha sido uno de los más afectados por el conflicto armado en el país, tanto de carácter político como relacionado con el narcotráfico, por lo cual su población ha sido víctima de distintas formas de desplazamiento. Así, los datos permiten entender que la universidad y el PCSUC sirven a una población diversa cultural, social y étnicamente, históricamente afectada en su posibilidad de desarrollo y sus ámbitos de extensión y proyección social son en buena medida de carácter rural.

Como antecedentes investigativos de interés se reseñaron documentos institucionales relacionados con el tema: a) un estudio sobre cancelación adelantado previamente desde la Vicerrectoría de Cultura y Bienestar, y b) el Modelo de permanencia y graduación diseñado con apoyo de las distintas vicerrectorías de la Universidad.

El primero de estos, el "Informe final cancelación de semestre 2014-2016" (Unicauca, 2017b) analiza los datos recogidos tanto del Sistema para la Prevención de la Deserción en las Instituciones de Educación Superior (Spadies), el cual dirige el Ministerio de Educación Nacional de Colombia (MEN), como los propios datos registrados por la Vicerrectoría de Cultura y Bienestar, ante las cancelaciones formales, bien sean de manera temporal o definitiva, que entre 2014 y 2016 hicieron estudiantes de distintos programas de la Universidad del Cauca.

El estudio revisó variables de tipo vocacional, académico, laboral, familiar, económico, psicológico, personal y de salud. Sus resultados (presentados de manera agregada para la universidad, y desagregada solo a nivel de facultades) permiten identificar que las variables académicas, económicas y vocacionales (en ese orden) son las que más inciden como causa de la cancelación del semestre.

El segundo documento es el "Modelo de permanencia y graduación estudiantil" (Unicauca, 2017c) parte del principio del modelo de Bienestar Universitario promovido desde el MEN, que demanda que las universidades realicen la labor de identificar y hacer seguimiento a las variables asociadas a la deserción y a las estrategias orientadas a disminuirla, propiciando especialmente estrategias con enfoque preventivo e integral. 
El modelo parte del análisis de datos de deserción de estudiantes de los distintos programas y facultades de la universidad y establece relaciones entre variables de orden económico, académico, social y psicológico. Igualmente, recoge las experiencias exitosas de modelos instaurados en otras universidades públicas y privadas de Colombia, estableciendo como objetivo "mejorar los índices de permanencia y graduación de los estudiantes de la Universidad del Cauca a través de procesos de apoyo integral que garanticen la continuidad y culminación exitosa de sus estudios universitarios" (Unicauca, 2017c).

Además de identificar el contexto institucional relacionado con el tema (y teniendo en cuenta que la investigación realizada en el PCSUC no se enfoca exclusivamente en la deserción sino en general en condiciones de vulnerabilidad de los estudiantes) fue necesario revisar el concepto de "vulnerabilidad estudiantil", en la medida en que atraviesa la perspectiva de abordaje la pesquisa. Al respecto, Ruiz (2012) señala que la vulnerabilidad se ha entendido a partir de diferentes y variados elementos como el riesgo, el estrés, la susceptibilidad o la adaptación, pero siempre definido en relación con algún tipo de amenaza. A los individuos o grupos sociales se los califica como vulnerables ante dichas amenazas, las cuales pueden ser de origen físico o socioeconómico.

Sánchez \& Egea (2011) señalan que, si bien la vulnerabilidad surge como concepto del seno de las ciencias ambientales frente a las afectaciones sociales de los riesgos naturales, más recientemente se destaca la importancia de la dimensión estructural de la vulnerabilidad sociodemográfica, como resultado del modelo de desarrollo, de la construcción social basada en la desigualdad y la desprotección. Pizarro (2001), enfocado específicamente en la vulnerabilidad social, devela cómo aunque esta se tiende a identificar con la pobreza, no es necesariamente la falta de ingresos la única causa de inseguridad e indefensión. Para el autor, si bien existe una dimensión objetiva de la vulnerabilidad, en la que se destacan los factores de trabajo, capital social (salud y educación) y relaciones sociales, también hay una dimensión subjetiva:

\footnotetext{
parece haber aumentado un sentimiento de indefensión en las capas medias y de bajos ingresos generado por el repliegue del estado de la función protectora que tuvo en el pasado e incluso por la preponderancia que han adquirido los valores que fomentan el esfuerzo individual en la lucha por la vida por sobre las lógicas colectivas" (Pizarro, 2001, p. 10).
}

Así, para el mencionado autor, es clave tener en cuenta que en Latinoamérica las sociedades tienden a la privatización de servicios de protección, la informalidad laboral y el fomento del consumo, como elementos implicados en una mayor vulnerabilidad de individuos y grupos, especialmente los de bajos y medios ingresos.

En cuanto a la vulnerabilidad estudiantil particularmente, tanto en los estudios académicos como para el MEN, esta aparece ligada a la deserción como su consecuencia. En un estudio diagnóstico sobre el tema, el MEN (2009) recuerda el enfoque de las IES como un sistema social con su estructura y valores, indicando que bajos niveles de integración aumentan la posibilidad de ocurrencia de la deserción; desde otras perspectivas también señala variables como las académicas, las socioeconómicas y las institucionales. Al conjugar esos y otros 
antecedentes en el enfoque, como el de Vincent Tinto, se define la deserción como "una situación a la que se enfrenta un estudiante cuando aspira y no logra cumplir su proyecto educativo" (MEN, 2009, p 22).

Puede decirse que la vulnerabilidad de los estudiantes ha sido estudiada en relación con sus condiciones económicas, trayectorias socioculturales y rasgos psicológicos, así como las características institucionales, todo lo cual confluye para generar distintas reacciones ante riesgos y amenazas del proceso formativo.

Partiendo de la revisión señalada, para efectos de esta investigación se concibió la vulnerabilidad como el grado de susceptibilidad que tienen las personas o grupos de ser afectados, amenazados, presionados o agredidos, por las condiciones provenientes de su entorno familiar, social, económico y cultural, o por ser portadores de un rasgo distinto al de la mayoría de las personas que le rodean (una orientación sexual, una etnia, una condición física o social distinta a las de la mayoría).

\section{Diseño metodológico}

La investigación se desarrolló con métodos cuantitativos y cualitativos (al combinar las técnicas de grupos de discusión y la encuesta) y su alcance es de tipo descriptivo. El diseño de los grupos focales incluyó tres preguntas suscitadoras sobre seis temas de discusión: a) pertenencia de los estudiantes a población LGBTIQ+, b) minorías étnicas, $c$ ) madres o padres cabeza de familia, $d$ ) discapacidad física o cognitiva, $e$ ) adicciones y f) problemas financieros.

El formulario de encuesta consta de 49 preguntas cerradas, las cuales se distribuyeron en cinco capítulos, respondiendo a las categorías de investigación: a) características sociodemográficas, b) condiciones de la vivienda, c) condiciones de manutención, d) condiciones de vulnerabilidad física o emocional y e) conocimiento y uso de servicios para estudiantes.

Este artículo presenta únicamente los resultados del análisis de las 125 encuestas aplicadas a estudiantes del PCSUC matriculados en los distintos semestres en curso durante el desarrollo de la investigación, esto es, en el segundo semestre de 2017. Debe señalarse que las encuestas se aplicaron durante sesiones ordinarias de clase con cada uno de los cuatro grupos en curso (semestres II, I, VI y VIII del PCSUC), buscando recoger información no con una muestra, sino con el universo de los estudiantes matriculados. No obstante, teniendo en cuenta que varios de los matriculados son estudiantes que ven solo algunas asignaturas o incluso solo están desarrollando el trabajo de grado y no asisten a clases, la suma total de 125 encuestas obtenidas representó un 55\% de los estudiantes formalmente matriculados de acuerdo con datos de la División de Registro Académico.

Los cuatro grupos de discusión se realizaron posteriormente a las encuestas, con estudiantes de cada uno de los semestres en curso, previo el inicio de una clase, y solicitando 
aleatoriamente la participación voluntaria de 8 a 12 personas interesadas en el debate de las categorías de análisis ya reseñadas. Para la sistematización de la información recogida en las encuestas, se utilizó el programa SPSS y para la graficación de los resultados, el MS Excel. Para el análisis de los grupos focales se transcribieron las intervenciones de los estudiantes participantes en cada uno de los cuatro grupos, y con dicha transcripción se realizó un reordenamiento de los datos a partir de los seis temas tratados y de las recomendaciones sugeridas por ellos hacia la Universidad en general, hacia la Vicerrectoría de Cultura y Bienestar, hacia la Facultad y hacia el PCSUC.

\section{Caracterización: ¿quiénes son nuestros estudiantes?}

Dadas las limitaciones de espacio del artículo, esta se limita a la descripción de un panorama parcial de los resultados obtenidos con la investigación, específicamente la caracterización derivada de la encuesta, dejando de lado extensos y significativos detalles obtenidos en los grupos de discusión.

\subsection{Vulnerabilidad socioeconómica: condiciones de los estudiantes encuestados}

Los 125 estudiantes encuestados se distribuyen de manera similar en relación con el sexo (gráfico 1), siendo ligeramente mayor la proporción de mujeres $(54 \%)$ que de hombres (46\%). En cuanto la edad (gráfico 2), el 79\% tiene entre 17 y 22 años, siendo predominante el rango de quienes tienen 19 a 20 años, con un 36\%. No obstante, siendo un programa diurno, es significativa la presencia de un $8 \%$ de estudiantes mayores de 25 años (edad en la que usualmente se ha alcanzado la titulación universitaria) o el estudiante universitario es trabajador en el día y estudia de noche.

Gráficos 1 y 2: Sexo y edad de los estudiantes.
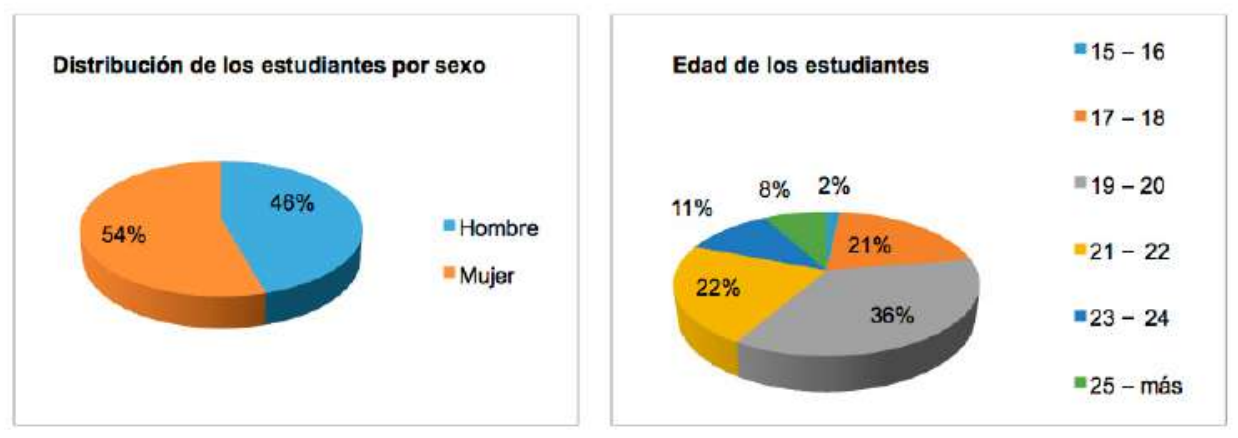

Fuente: Elaboración propia.

Los estudiantes del PCSUC son, en su mayoría, originarios del Cauca (gráfico 3), pero solo un poco más de la mitad, el 55\%, son de Popayán, mientras el 14\% proviene de otros municipios caucanos. Casi una tercera parte, el 31\% proviene del resto del país, siendo un $22 \%$ de ellos nacidos en el Huila, Nariño o el Valle, lo que muestra 
el impacto regional del PCSUC. Debe señalarse que dicho origen (con frecuencia) es también rural, lo que imprime características adicionales al perfil del estudiante promedio.

Gráfico 3: Lugar de nacimiento de los estudiantes.

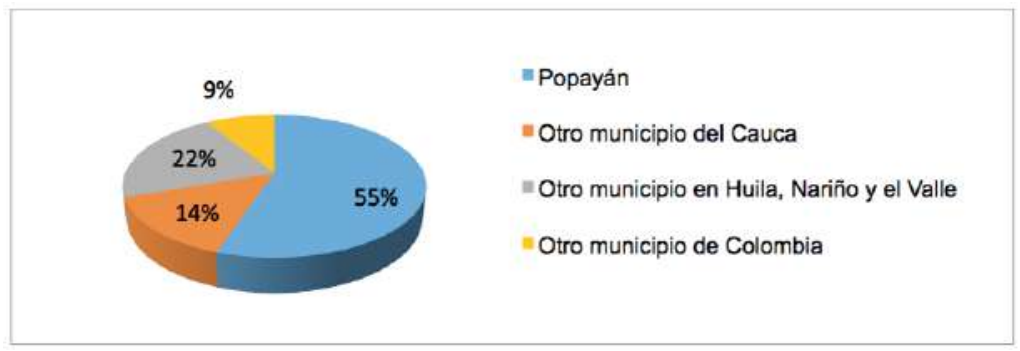

Fuente: Elaboración propia.

Es importante señalar que el motivo de traslado familiar (gráfico 4) de aquellos estudiantes (oriundos de otros municipios distintos a Popayán) es básicamente la búsqueda de oportunidades laborales $(15.2 \%)$ y oportunidades educativas $(13.6 \%)$, lo que ilustra la influencia que tiene Popayán como ciudad universitaria en la región. Además, de ese $31 \%$ de estudiantes que provienen de zonas distintas al Cauca, un $4 \%$ ha migrado a causa del desplazamiento forzado, lo cual implica un compromiso del PCSUC en general, frente a este segmento de estudiantes cuya condición amerita un mayor respaldo y seguimiento psicosocial, especialmente en el contexto del actual proceso de construcción de paz.

Gráfico 4: Motivo de traslado familiar a Popayán.

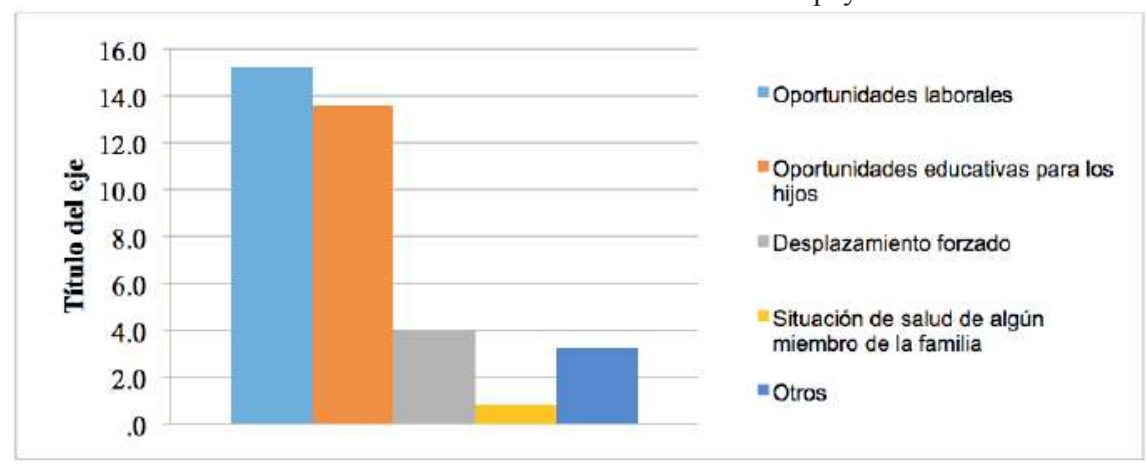

Fuente: Elaboración propia.

Los estratos de las viviendas de residencia de los estudiantes del PCSUC (gráfico 5) son en su mayoría los estratos 3 y 2, sumando entre ellos el $62 \%$. El 23\% vive en viviendas de estrato 4, una no menos importante proporción del $12 \%$ reside en estrato 1 y solo un $2 \%$ en estrato 5 . Lo anterior describe al promedio de estudiantes del PCSUC como un estudiante de estrato medio bajo. Sin embargo, la gran mayoría de las viviendas 
de residencia de los estudiantes (gráfico 6), por encima del 90\%, cuentan con todos los servicios básicos (acueducto, alcantarillado y energía) y con Internet, servicio fundamental para los estudiantes del PCSUC.

Gráficos 5 y 6: Estratos de la vivienda de residencia y disposición de servicios en la vivienda de residencia.

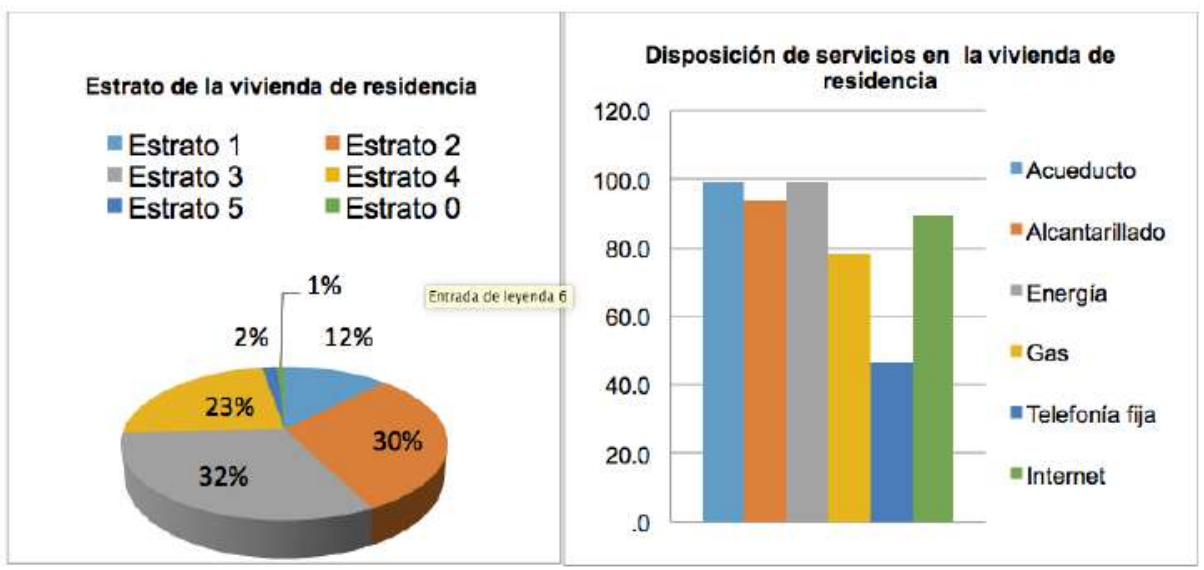

Fuente: Elaboración propia.

En la gran mayoría de los casos (72\%) los estudiantes cuentan con el respaldo de su familia para el pago de las matrículas (gráfico 7), aunque un 15\% dice que la fuente de financiación de las mismas varía cada semestre y una cifra destacable del $8 \%$ son estudiantes que trabajan para pagar la matrícula. Para su manutención, tres cuartas partes de los encuestados afirmaron también que son sus familias quienes les garantizan los gastos respectivos (gráfico 8) y un 14\% señala tener para ello fuentes variables cada semestre.

Gráficos 7 y 8: Origen del pago de matrícula y origen de la manutención.

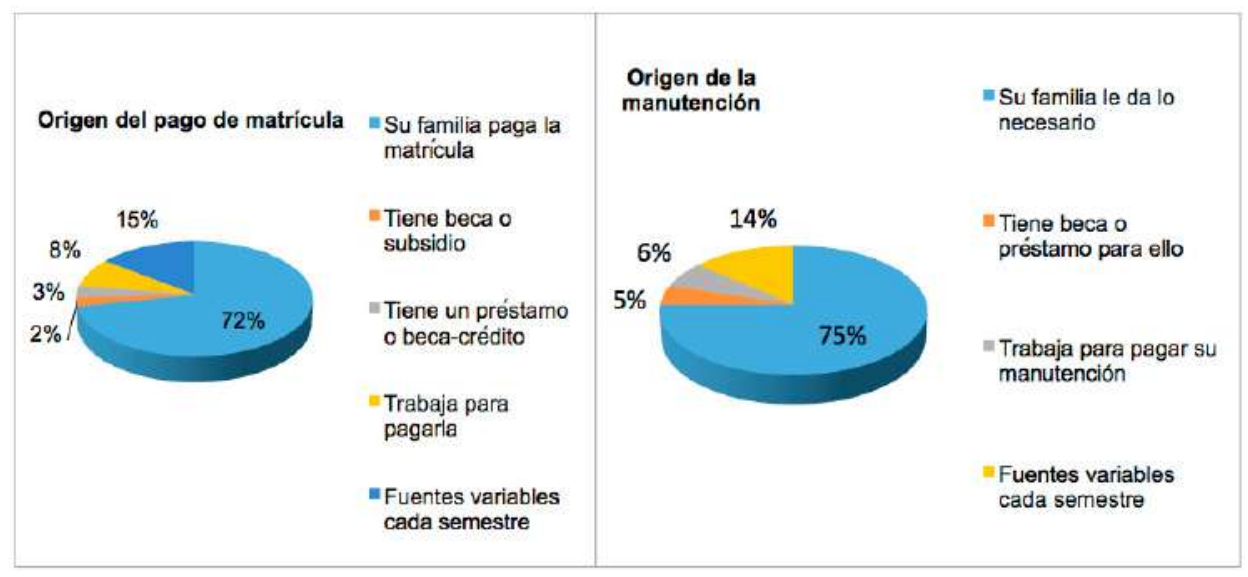

Fuente: Elaboración propia. 
Pese al respaldo familiar (y teniendo en cuenta los estratos de residencia de los estudiantes) una proporción significativa no tiene una óptima alimentación (gráfico 9), ya que muchos de ellos no consumen de manera adecuada las tres comidas diarias, sino que obvian alguna de ellas, o la consumen de manera incompleta.

Gráfico 9: Características de la alimentación diaria.

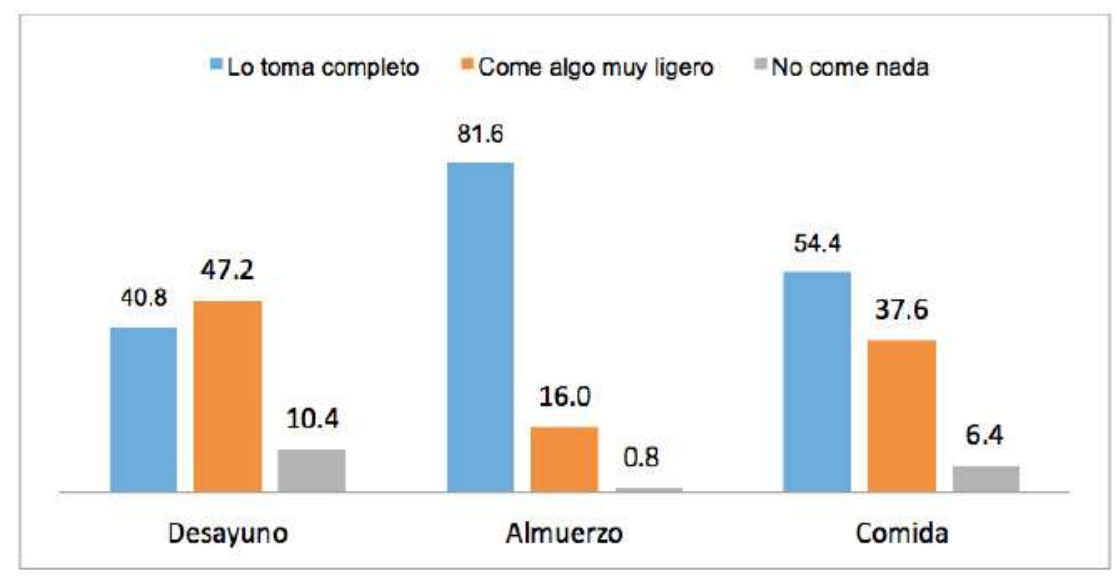

Fuente: Elaboración propia.

En el caso del desayuno, cerca de la mitad de los estudiantes $(47.2 \%)$ dicen comer algo muy ligero y un $40 \%$ señala comerlo completo, mientras un $10 \%$ no come nada en la mañana, lo cual implica condiciones inadecuadas de alimentación si se tiene en cuenta los cursos del PCSUC se distribuyen en horarios matutinos y vespertinos. El almuerzo en su gran mayoría es consumido de manera completa $(81.6 \%)$, aunque un importante $16 \%$ dice hacerlo de manera ligera. Y en cuanto a las cenas, si bien algo más de la mitad de los encuestados dice tomar la comida completa, una importante proporción del $37.6 \%$ cena algo ligero mientras un $6.4 \%$ no come nada.

Vale decir que las cifras (en cuanto a alimentación) deben ser tomadas en consideración con la reserva pertinente, toda vez que la pregunta por si la comida es ligera o completa es subjetiva, partiendo de la percepción individual de lo adecuado, que da pie a interpretaciones que dependen de la condición material y los hábitos del estudiante. Así por ejemplo, aquello considerado como una "comida completa", puede no ser lo adecuado en términos nutricionales. 
Gráfico 10: Tipo de alimentación según estrato de vivienda del estudiante (desayuno).

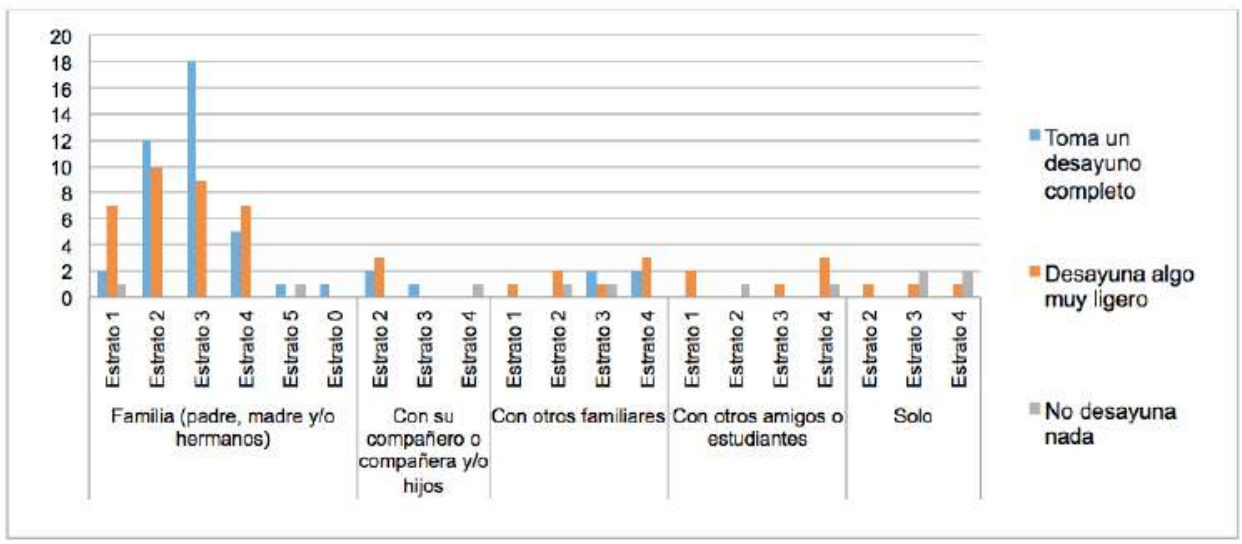

Fuente: Elaboración propia.

Al cruzar esta variable de alimentación con los estratos de residencia (gráfico 10) y con el tipo de cohabitación, se observa que los estudiantes residentes en estrato 4 , tienden a desayunar algo ligero o no desayunar nada, independientemente de con quién vivan. Igualmente para todos los estratos, quienes viven solos o con otros amigos o estudiantes, tienden a desayunar algo muy ligero o a no hacerlo. Quienes desayunan completo son preferentemente quienes viven con sus familias en todos los estratos, con excepción del estrato 1, así como quienes viven con otros familiares, en el caso de estratos 3 y 4.

Se encontró igualmente que el comportamiento frente a la alimentación (en el caso de la toma del almuerzo y la cena) no varía significativamente a lo anotado frente al desayuno, en relación con el estrato de la vivienda y las personas con quienes residen los estudiantes. Puede decirse que la tendencia general es a tomar el almuerzo completo y descuidar (en primer lugar) el desayuno y (en segundo) la comida, sin mayor relación con el tipo de hogar de los estudiantes, con la salvedad de que es más común el tomar completas las comidas si se convive con familiares, lo cual constituye un factor protector.

\subsection{Vulnerabilidad sociocultural e institucional: pertenencia a una población potencialmente vulnerable}

En segunda instancia, se presentan los resultados sobre el tema específico de las seis variables estimadas como posibles causas de vulnerabilidad e identificadas a partir de la aproximación cotidiana a la realidad de los estudiantes. Al preguntar por la pertenencia a una de las seis categorías de población consideradas potencialmente vulnerables, en su gran mayoría los estudiantes del PCSUC afirmaron no pertenecer a ellas, pero proporciones significativas que varían entre un $4 \%$ y un $17.6 \%$ sí se reconocieron como pertenecientes a ellas. Entre estos (gráfico 11), los más significativos son en su 
orden: el 17.6\% que admiten ser consumidores de alcohol, el 13.6\% que se reconocen como población LGBTIQ+ y el $12.8 \%$ que pertenecen a minorías étnicas, básicamente indígenas y afrodescendientes.

Gráfico 11: Pertenencia a una población potencialmente vulnerable.

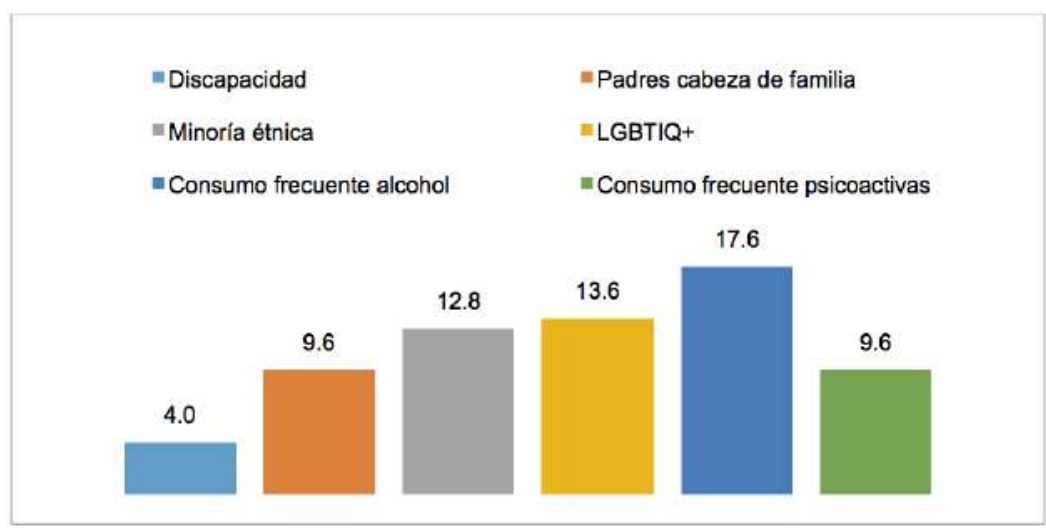

Fuente: Elaboración propia.

Entre los pocos estudiantes en condición de discapacidad ${ }^{3}(4 \%)$, un poco más de la mitad de ellos considera que han sido discriminados y ello ha afectado su rendimiento académico. De manera adicional, la mayoría de los estudiantes del PCSUC (92\%) estimaron que los espacios físicos de la universidad no han sido adecuados para personas en condición de discapacidad, lo cual significa que existe una triple vulnerabilidad: en primera instancia, la inherente a la discapacidad misma, en segundo lugar, aunque en muy baja proporción, la discriminación de la que son objeto por parte de distintos miembros de la comunidad universitaria y, por último, la inadecuación de los espacios en los que desarrollan su proceso formativo.

En relación con la pertenencia a minorías étnicas, $12.8 \%$ de los estudiantes respondieron sentirse pertenecientes a una comunidad indígena o a una afrodescendiente. De ellos, solo uno afirmó haber sido víctima de discriminación por ello. En el caso de la población LGBTIQ+, se identificaron como pertenecientes el 13.6\% de los encuestados, pero sólo uno admitió haber sido víctima de discriminación ${ }^{4}$. La discriminación también se validó al indagar a la totalidad de los estudiantes acerca de si han sido testigos de discriminación a otros estudiantes a causa de su condición de discapacidad, su pertenencia a una minoría étnica o por su orientación sexual (gráfico 12). Más de una tercera parte de ellos (37\%) afirmó que sí.

3 La condición de discapacidad se definió a los encuestados como una limitación o dificultad innata o adquirida, de carácter permanente de tipo motor, visual, auditiva o aún cognitiva.

4 Este dato contrasta de manera notable con la información recogida en los grupos focales, según la cual sí hay varios casos especialmente de estudiantes del PCSUC discriminados por su orientación sexual, especialmente por parte de otros estudiantes. 
Gráfico 12: ¿Han sido testigos de discriminación a estudiantes por razón de su discapacidad, etnia u orientación sexual?

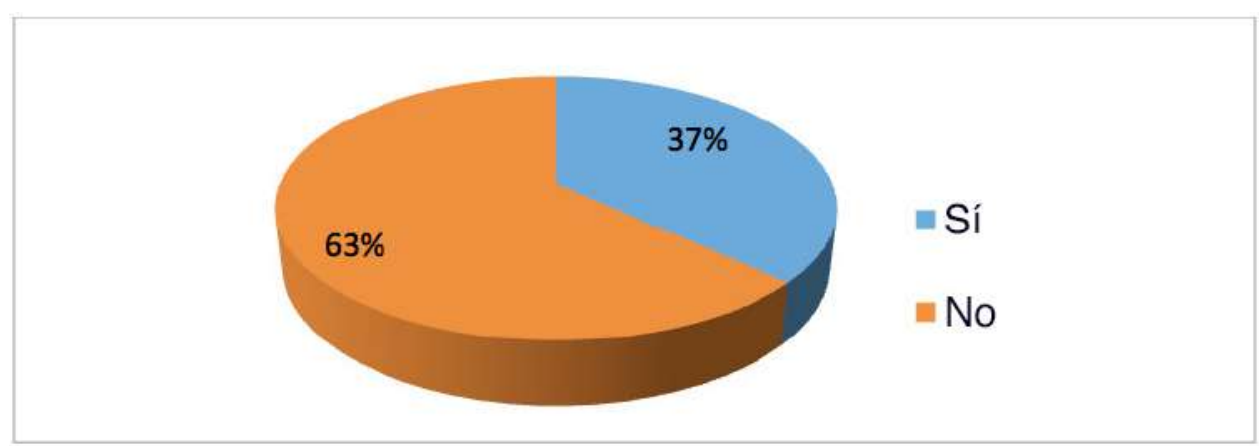

Fuente: Elaboración propia.

Además es importante que la mayoría reconoce al estamento estudiantil (de distintos programas académicos), como los principales actores universitarios de dicha discriminación (gráfico 13), seguidos en mucha menor medida por los docentes, lo cual muestra un ambiente hostil para el desarrollo de la formación académica de estudiantes con discapacidad, pertenecientes a una minoría étnica o a la población LGBTIQ+.

Gráfico 13: Actores universitarios de la discriminación a estudiantes.

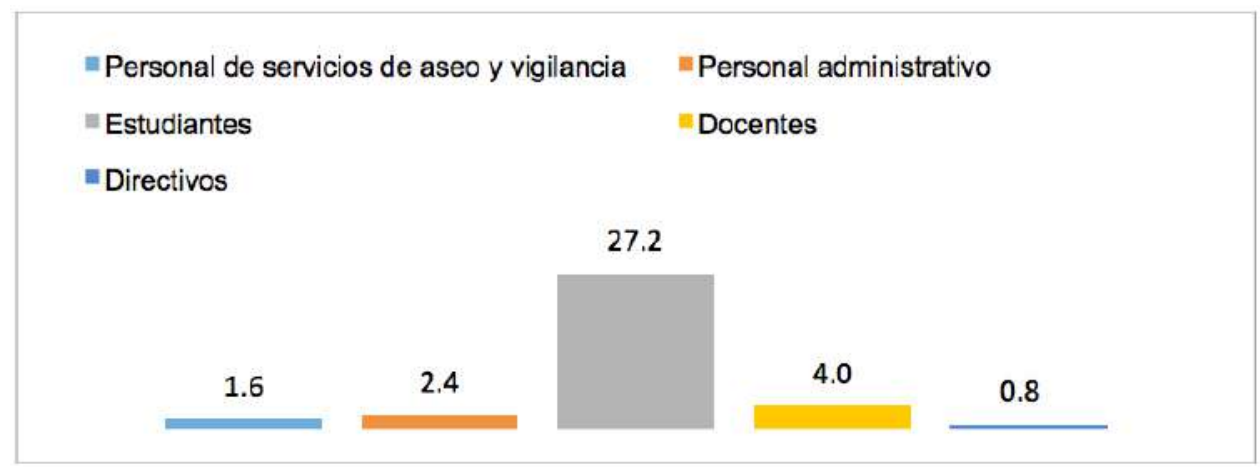

Fuente: Elaboración propia.

Frente a otras condiciones que pueden constituir causa de vulnerabilidad estudiantil (al generar dificultades en el proceso formativo) el 9.4\% de los estudiantes (que son padres de familia) en su mayoría estiman que su condición ha sido obstáculo para un óptimo rendimiento académico y que, por lo tanto, requieren un tratamiento especial por parte de profesores y directivos, a fin de facilitar el desempeño de ambos roles de manera adecuada. La mayoría de los 12 casos de estudiantes del PCSUC (que son padres de familia) son mujeres y se ubican preferentemente en los rangos de edad más altos (gráfico 14), aunque el PCSUC existen tres casos de jóvenes entre 17 y 20 años que ya son padres cabeza de familia. 
Gráfico 14: Estudiantes padres y madres cabeza de familia según edad.

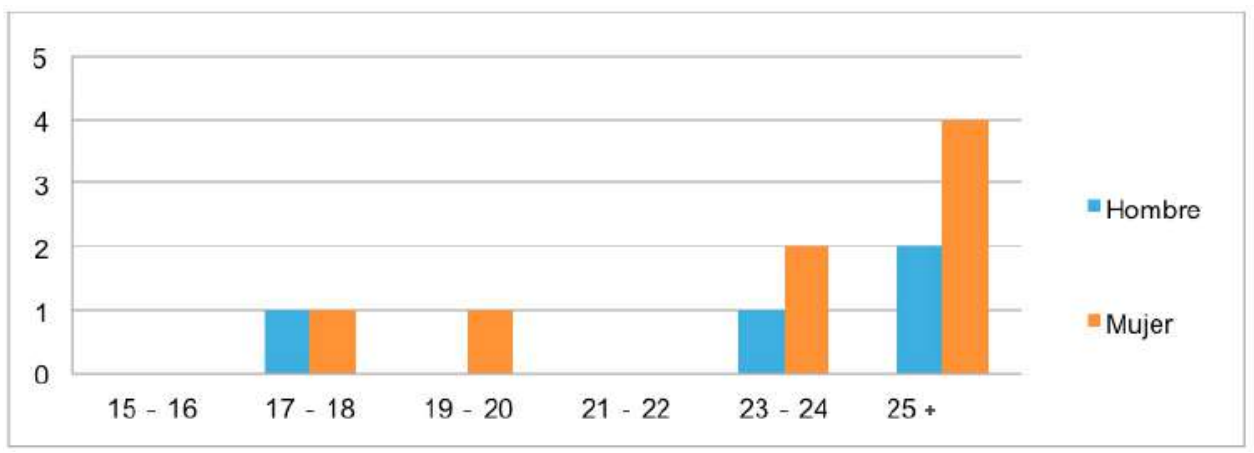

Fuente: Elaboración propia.

Igualmente se indagó a cada encuestado sobre por la posible condición de adicción de otros estudiantes. En esta pregunta sobre terceros, igualmente la subjetividad debe tenerse en cuenta, en tanto las perspectivas sobre lo que significa ser un adicto o ser un consumidor ocasional, varían en cada persona. No obstante, la pregunta se hizo justamente para identificar la percepción al respecto (gráfico 15). Aunque una tercera parte, el 32.8\% de los estudiantes del PCSUC dicen no conocer casos de compañeros con adicciones, el 67.2\% identifica al menos un caso. De ellos, la mayoría afirma conocer varios casos: un $29.6 \%$ dice que conoce uno o dos casos, un $17.6 \%$ dice que conoce de tres a cinco casos y un importante porcentaje del $12.8 \%$ afirma que conoce muchos casos.

Gráfico 15: Conocimiento de casos de estudiantes con adicciones.

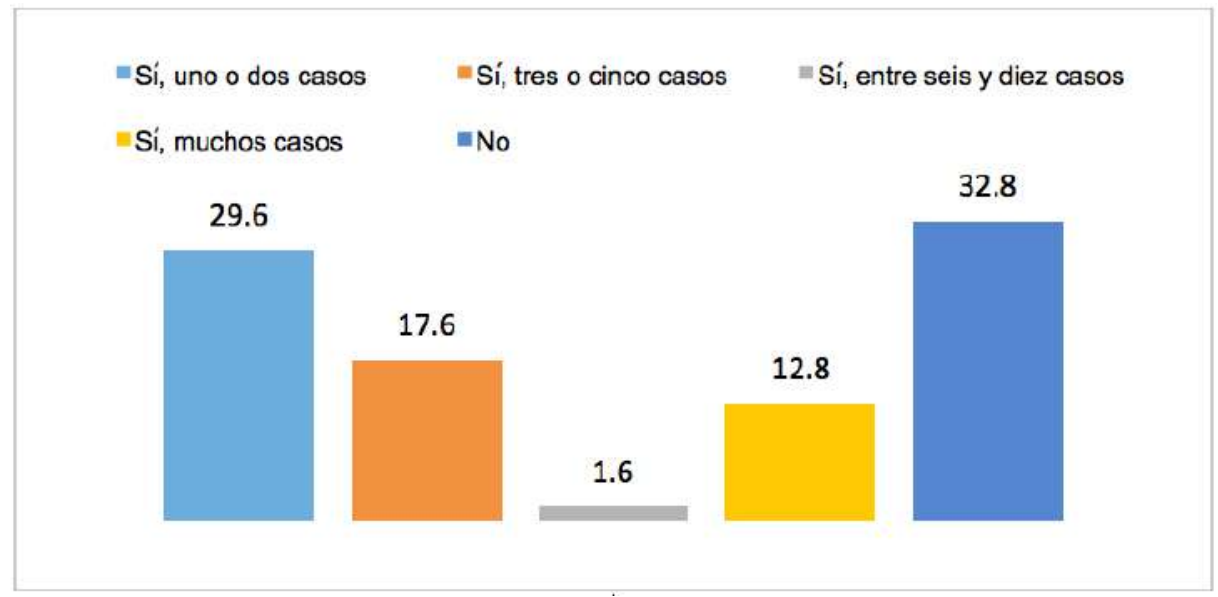

Fuente: Elaboración propia. 
En contraste, al preguntar a cada encuestado si sus compañeros de estudios eran consumidores habituales $^{5}$ de alcohol o sustancias psicoactivas (gráfico 16), las proporciones bajaron significativamente: quienes se reconocen a sí mismos como consumidores habituales de alcohol (17.6\%) son el doble de los estudiantes que admiten ser consumidores habituales de sustancias psicoactivas (9.6\%), lo cual puede estar ligado a la mayor aceptación social y cultural del consumo de alcohol. No obstante, la casi totalidad de quienes se asumieron como "consumidores habituales", afirman no sentirse afectados en su rendimiento académico (gráfico 17) debido al consumo de dichas sustancias, y en general tampoco consideran que requieran algún tipo de ayuda o atención institucional.

Gráficos 16 y 17: Consumidores habituales de alcohol o sustancias psicoactivas y consideración de afectación del rendimiento por el consumo.

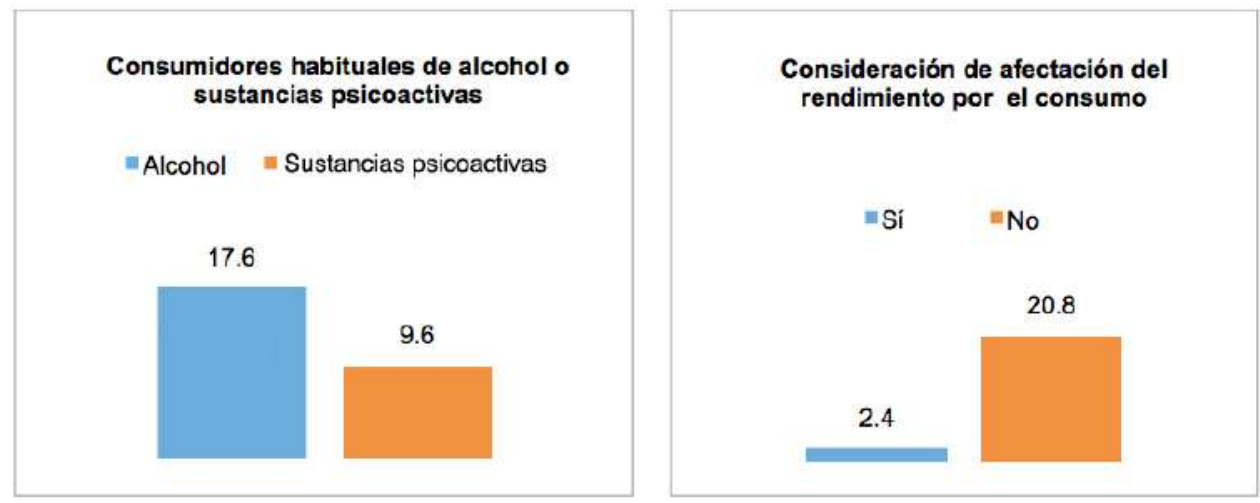

Fuente: Elaboración propia.

Al cruzar los consumos con los rangos de edad y por sexo, se encuentran coincidencias con el relato obtenido en los grupos focales, en el sentido de que hay un momento de la formación en la que los estudiantes actúan descontroladamente frente a ciertos comportamientos que antes les fueron vedados, como el consumo de licor. Se observa así que los rangos de edad intermedios son los que concentran el mayor número de consumidores habituales de alcohol y de sustancias psicoactivas, siendo en general más los casos de hombres que de mujeres.

\section{Conclusiones}

De acuerdo con el diagnóstico del MEN (2001), “el principal factor determinante del abandono de estudios en Colombia se sitúa en la dimensión académica: está asociado al potencial o capital cultural y académico con el cual ingresan los estudiantes a la educación superior. Los factores financieros y socioeconómicos están a continuación, seguidos por los institucionales y los de orientación vocacional y profesional” (p. 10).

5 Estimando consumo habitual como "el hecho de utilizarlas al menos una vez por semana" 
Los resultados de la investigación (parcialmente presentada) permitieron identificar que en el caso del PCSUC, son los factores financieros, socioeconómicos e institucionales, los que más generan amenazas al proceso formativo y que de las variables estudiadas, tres son las que representan mayor vulnerabilidad estudiantil:

- Las dificultades económicas de los estudiantes, dado que es generalizada y desatendida, en la medida que se ha naturalizado como inherente a la mayoría del estudiantado de la universidad pública.

- La referida a condiciones de salud como discapacidad motriz, sensorial o cognitiva, así como enfermedades de salud física o mental que no siempre son evidentes para los docentes y compañeros.

- La condición de padre o madre cabeza de familia, que implica duplicidad de compromisos y responsabilidades por parte de dichos estudiantes.

La pertenencia a una minoría étnica o a la población LGBTIQ+ no son percibidas por los estudiantes en general como situaciones de vulnerabilidad. No obstante, sobre las minorías étnicas se destaca una percepción de apoyo y estímulo, mientras frente a la población estudiantil LGBTIQ+ se da cuenta de significativas proporciones de discriminación por parte del estudiantado, especialmente de aquellos pertenecientes a los otros programas de la Facultad. Frente a ello, parece existir un factor protector que genera mayor resiliencia y es el respaldo interno dentro del PCSUC, por parte de la mayoría de estudiantes y docentes, el cual se percibe en un trato igualitario y respetuoso de su orientación sexual. En cuanto a la tendencia a las adicciones al consumo de alcohol o psicoactivos, en general no se considera una condición de mayor vulnerabilidad ${ }^{6}$, por parte de los mismos consumidores, quienes no se muestran interesados por transformar dicha realidad.

Pese a las distintas percepciones, se considera que la Universidad, la Facultad y el PCSUC, en particular, les cabe la responsabilidad institucional de prestar mayor atención a todos los casos y buscar alternativas de apoyo y atención a los estudiantes, en aras de mejorar el bienestar del entorno de formación. Debe señalarse que (más allá de los resultados de las encuestas aquí presentados, de acuerdo con la información recolectada en los grupos de discusión), hay otras dificultades para el proceso de formación estudiantil que inciden en su rendimiento académico, impactando a la larga en casos de repitencia, inasistencia o deserción. Las más importantes de ellas según la percepción de los estudiantes del PCSUC, son las que se listan a continuación, sin un orden de prioridad:

- Horarios de clase dispersos que dificultan las ya escasas opciones laborales y duplican los gastos diarios de transporte.

- Costos de matrícula elevados, frente a las condiciones económicas vigentes de los estudiantes y sus familias.

6 Sin embargo, de acuerdo con lo relatado en grupos focales, desde fuera, otros estudiantes no consumidores o consumidores poco habituales perciben desmejora en el rendimiento académico de aquellos que estiman son consumidores habituales. Dadas las condiciones de poca atención y desconcentración con la que a veces comparten el aula de clases y otros espacios de trabajo fuera del aula, estiman además que hay una afectación al grupo en general. 
- Altos gastos semestrales en materiales de lectura que deben ser fotocopiados.

- Ineficiente servicio de cafeterías universitarias, por la calidad de los alimentos que se ofrecen y por su costo.

- Falta de flexibilidad en el manejo de horarios y cumplimiento de compromisos hacia los estudiantes padres y madres cabeza de familia.

- Estigmatización generalizada de parte de estudiantes de otros programas hacia el PCSUC por su ambiente de respeto a la diversidad sexual.

- Ausencia de mecanismos de adaptación en espacios físicos y dinámicas institucionales para facilitar el desarrollo académico de estudiantes con discapacidad o con enfermedades físicas o mentales que afectan su proceso de formación.

- Naturalización y ausencia de estrategias preventivas y de disuasión frente al consumo de alcohol y sustancias psicoactivas.

- Desconocimiento de las bondades de los servicios de salud y formación integral, así como de los mecanismos de participación estudiantil, como instancias que propician una mejora en la calidad de vida y el entorno de formación del estudiante.

\section{Recomendaciones}

A partir de los hallazgos identificados, surgen algunas recomendaciones tendientes a disminuir las causas de vulnerabilidad estudiantil en el PCSUC, las cuales podrían considerarse a nivel institucional como extensivas hacia otros programas:

- Se considera fundamental desarrollar una estrategia o protocolo de seguimiento y evaluación periódica para la detección de casos estudiantiles específicos de vulnerabilidad, con el fin de atender de manera oportuna las situaciones particulares.

- Por parte del PCSUC, se debe privilegiar (en el diseño de los horarios de clase) la concentración de bloques matutinos o vespertinos de las distintas actividades presenciales, para facilitar la distribución concentrada, a fin de disminuir gastos de transporte y favorecer la oportunidad de trabajo a estudiantes de escasos recursos

- Teniendo en cuenta que las realidades materiales de los estudiantes y sus familias es variable, se sugiere diseñar una estrategia de actualización de la condición económica de aquellos estudiantes que soliciten expresamente el estudio de su situación a finales de semestre. Con ello, ante la Vicerrectoría Académica podrían tramitarse las solicitudes respectivas, revisar los documentos que sustentan la reducción de sus ingresos y conceder ( $\mathrm{si}$ es el caso) la disminución en el valor de las matrículas de manera coherente con su realidad.

- Mejorar la calidad de la oferta nutricional de las cafeterías y el costo de los alimentos para los estudiantes, especialmente para aquellos de bajos recursos, debe ser una prioridad institucional. Revisar la opción de desayunos y almuerzos subsidiados, así como la expedición de un carnet semestral de auxilio económico, que podría ser una solución ligada a la identificación semestral de casos de estudiantes con ingresos reducidos. Una medida adicional y sencilla es la provisión de uno o dos hornos microondas en la cafetería de la Facultad, para facilitar el consumo de alimentos de estudiantes que llevan desde su casa alguna preparación. 
- Se sugiere estudiar la viabilidad financiera de un centro de fotocopiado en la Facultad, en el cual reposen varias copias de cada material de lectura necesario para el desarrollo de los cursos. Dichas copias pueden ser ofrecidas en calidad de préstamo por uno o dos días, mediante carnet estudiantil, del mismo modo que los prestamos bibliotecarios.

- Con los casos de padres y madres cabeza de familia, desde la coordinación del PCSUC se pueden recibir solicitudes expresas para hacer seguimiento de las situaciones particulares de cada uno y las necesidades que de estas se derivan, a fin de solicitar a los docentes respectivos, la concertación de condiciones de flexibilidad en la llegada o el retiro de las horas de clase.

- En el mismo sentido, el mecanismo de solicitud expresa hacia la coordinación del PCSUC puede utilizarse para considerar los casos especiales de salud, es decir, de estudiantes en condición de discapacidad o con enfermedades físicas o mentales que requieren trato especial, para tenerles en cuenta en la asignación de espacios adecuados a su situación (de acceso, iluminación, amplitud, entre otros). Dicha asignación de espacios deberá ser coordinada y priorizada por la Facultad para todos los estudiantes de los distintos programas. En todo caso, esto no exime a la Universidad de su responsabilidad de adecuar sillas sube-escaleras en los accesos al segundo piso del claustro colonial, que es sede de la Facultad y constituye patrimonio arquitectónico.

- En torno a la discriminación y estigmatización de los estudiantes del PCSUC como personas LGBTIQ+, es necesario que (desde la Vicerrectoría de Cultura y Bienestar y desde la propia Facultad) se generen estrategias de sensibilización hacia el respeto de la diversidad sexual. Del mismo modo que se han promovido y apoyado colectivos de organización indígena (como el "Cabildo Indígena Universitario") o afrodescendientes (Como el "Palenque Universitario") se debe promover una comunidad estudiantil LGBTIQ+ que apoye la tarea de visibilizar y sensibilizar a toda la comunidad universitaria hacia el respeto de la diversidad sexual.

- Programas de prevención y protección ya existentes (en la Vicerrectoría de Cultura y Bienestar) deben fortalecerse y difundirse hacia el PCSUC con mayor eficiencia para acercar a la población estudiantil al debate y conocimiento de riesgos y alternativas a la adicción. La oferta periódica de información (en forma de talleres, charlas y debates en la Facultad) puede aportar a una mayor sensibilización sobre el problema del consumo permanente de alcohol, tabaco y sustancias psicoactivas. Simultánea y complementariamente, la mayor promoción y disposición de espacios alternativos (como los colectivos artísticos o la formación deportiva) se considera importante para ofrecer a los estudiantes del PCSUC otras maneras de mejorar su entorno formativo.

- Propiciar un espacio semanal de debate sobre todos los temas de este estudio, se considera viable y pertinente como complemento a la formación académica. Se trataría de un taller permanente de bienestar estudiantil, en el que (a través de charlas, discusiones abiertas y talleres) se trabajen los temas para ponerlos cotidianamente en la agenda, asignándoles la importancia que tienen para generar mayor sensibilidad y conciencia al respecto. 


\section{Fuentes consultadas}

Ministerio de Educación Nacional de Colombia, MEN (2009). Deserción estudiantil en la educación superior colombiana. Metodología de seguimiento, diagnóstico y elementos para su prevención. Recuperado de https://www.mineducacion.gov.co/ sistemasdeinformacion/1735/articles-254702_libro_desercion.pdf

Pizarro, R. (2001). La vulnerabilidad social y sus desafios. Santiago de Chile: CEPAL. Recuperado de https://repositorio.cepal.org/bitstream/handle/11362/4762/ S0102116_es.pdf

Ruiz, N. (2012). La definición y medición de la vulnerabilidad social. Un enfoque normativo. Revista Investigaciones Geográficas, 77, 63-74. Recuperado de http:// www.scielo.org.mx/pdf/igeo/n77/n77a6.pdf

Sánchez, D. \& Egea, C. (2011). Enfoque de vulnerabilidad social para investigar las desventajas socioambientales. Su aplicación en el estudio de los adultos mayores. Revista Papeles de población, 17(69). Recuperado de http://www.scielo.org.mx/ scielo.php?script $=$ sci_arttext\&pid=S1405-74252011000300006

Universidad del Cauca, Unicauca (2017a). Informe de Autoevaluación con fines de acreditación de alta calidad, Programa de Comunicación Social. Informe técnico presentado en instancias universitarias y posteriormente al Ministerio de Educación Nacional de Colombia (MEN).

Universidad del Cauca, Unicauca (2017b). Informe final cancelación de semestre 20142016. Recuperado de http://facultades.unicauca.edu.co/vicecultura/sites/default/ files/informe_cancelacion_2014_-_2016_3.pdf

Universidad del Cauca, Unicauca (2017c). Modelo de permanencia y graduación estudiantil. Recuperado de http://facultades.unicauca.edu.co/vicecultura/ sites/default/files/documentos/politica_de_permanencia_y_graduacion_ estudiantil-21-12-17.pdf

Universidad del Cauca, Unicauca (2018). Estadística de Estudiantes pregrado y posgrado. Universidad del Cauca. Oficina de Planeación y Desarrollo Institucional. Fuente SIMCA - Fecha de corte 24-05-2019. Recuperado de https://app.powerbi.com/view?r=eyJr IjoiNGIzNDU5NGUtODUwNi00NDY0LWIzYWUtZGMyZTdkNWU2MD QyIiwidCI6ImU4MjE0OTM3LTIzM2ItNGIzNi04NmJmLTBiNWYzMz M3YmVIMSIsImMiOjF9 